\title{
DETERMINACIÓN DE Salmonella spp EN CENTROS DE BENEFICIO CLANDESTINO DE POLLOS DE ENGORDE EN LIMA, PERÚ
}

\author{
Determination of Salmonella SPP in Illegal Abattoirs of Broilers \\ in Lima, Peru
}

\author{
Hatzumi Zambrano F. ${ }^{1}$, Juan Lucas L. ${ }^{1,2}$, Miguel Vilca L. ${ }^{1,3}$, Daphne Ramos D. ${ }^{1}$
}

\section{Resumen}

El objetivo del presente estudio fue determinar la presencia de Salmonella spp en pollos de engorde en 17 centros de beneficio clandestino de Lima, Perú. Se colectaron muestras de superficie corporal, mediante el método de enjuague, y muestras de hisopado cloacal de 170 aves. El aislamiento e identificación de Salmonella spp se hizo mediante los procedimientos rutinarios de laboratorio. El 23.5\% de las muestras de superficie corporal y el $32.4 \%$ de muestras de hisopado cloacal fueron positivas a Salmonella spp, sin que hubiera diferencias entre centros de beneficio donde el proceso finaliza con el desplumado o donde finaliza con el eviscerado. El grado de concordancia de los resultados para ambos métodos de toma de muestra no fue significativo $(\mathrm{k}=0.074, \mathrm{k}=0.146)$, de allí que se requiere tomar ambos tipos de muestra para determinar la posible contaminación de la canal por Salmonella spp.

Palabras clave: Salmonella spp, centros de beneficio, canales de pollos, evisceración

\section{Abstract}

The aim of this study was to determine the presence of Salmonella spp in broilers from 17 illegal abattoirs in Lima, Peru. Samples were collected from the body surface of carcasses (using the rinsing method) and cloacal swabs in 170 broilers. The isolation and identification of Salmonella spp was done through conventional laboratory methods. Results showed $23.5 \%$ of body surface samples and $32.4 \%$ of the cloacal swabs were positive to Salmonella spp, without differences between abattoirs where evisceration

\footnotetext{
${ }^{1}$ Laboratorio de Salud Pública y Salud Ambiental, Facultad de Medicina Veterinaria, Universidad Nacional Mayor de San Marcos, Lima

${ }^{2}$ E-mail: jrlucas.pe@gmail.com

${ }^{3}$ E-mail:mvilcal@unmsm.edu.pe
}

Recibido: 13 de junio de 2012

Aceptado para publicación: 15 de marzo de 2013 
was or was not carried out. The degree of concordance of the results for both sampling methods was not significant $(\mathrm{k}=0.074, \mathrm{k}=0.146)$, indicating the both types of samples have to be taken to determine possible contaminations of the carcasses by Salmonella spp.

Key words: Salmonella spp, abattoirs, broilers carcasses, evisceration

\section{INTRODUCCIÓN}

La salmonelosis es una de las zoonosis con mayor frecuencia de reporte de brotes de transmisión alimentaria en el mundo, incluido los países desarrollados (Gutiérrez et $a l ., 2008)$, siendo la carne de ave y sus derivados una de las fuentes principales (FAO/ OMS, 2003). En el Perú, y en especial en Lima, la población tiene una demanda cada vez mayor de la carne de pollo, siendo actualmente la principal fuente proteica (27.6 $\mathrm{kg}$ por habitante al año), lo que se refleja en el crecimiento de la producción avícola (MINAG, 2010).

En el Perú, solo el 25\% de la producción avícola se beneficia en centros autorizados (Málaga, 2011), ya que la gran mayoría se sacrifica y comercializa en establecimientos clandestinos y en condiciones sanitarias deficientes. Las deficiencias detectadas como falta de agua, uso de agua contaminada, falta de medios de refrigeración, y mal proceso de eviscerado, entre otras, pueden conllevar a la contaminación de la canal con Salmonella spp. Es de esperarse que mejoras en las prácticas de higiene en estos centros de beneficio puedan reducir significativamente este riesgo (Heyndrickx et al., 2002).

Si bien Salmonella spp puede afectar a personas de cualquier edad, la información epidemiológica indica que la susceptibilidad es más alta en los infantes, ancianos y personas inmunocomprometidas (FAO/OMS, 2003). Por ello, existen normas sanitarias referentes al límite aceptable de Salmonella spp en alimentos, y en el Perú, la vigente Norma Técnica de Salud N ${ }^{\circ} 071$ establece la ausencia de Salmonella spp en $25 \mathrm{~g}$ de carne de ave (MINSA/DIGESA, 2008).

En el 2008, la Organización Panamericana de la Salud determinó que el $16 \%$ de aislamientos en casos de enfermedades transmitidas por alimento durante el periodo 2000 2008 en 10 países de Sudamérica, incluido el Perú, correspondió a Salmonella spp, de los cuales el $20 \%$ se presentó en carne de ave (WHO-GFN, 2008). Asimismo, en el 2010 se detectó un inusual aumento de casos de salmonelosis humana, en su mayoría pediátricos, en diversos hospitales de Lima, de los cuales dos tenían como fuente a productos cárnicos avícolas (Zamudio et al., 2011).

La vigilancia epidemiológica por parte de las autoridades de salud es necesaria para apreciar la magnitud del problema en cada país, así como para conocer el origen de los brotes y adoptar las medidas convenientes a fin de reducir los riesgos (Acha y Szyfres, 2003). El presente estudio tuvo como objetivo determinar la presencia de Salmonella spp en pollos de engorde de 17 centros de beneficio clandestino de Lima.

\section{Materiales y Métodos}

\section{Lugar de Estudio}

El catastro nacional de 2007 del Servicio Nacional de Sanidad Agraria (SENASA) del Perú incluye 177 centros de beneficio autorizados y no autorizados (Guerrero, 2007). En los centros de beneficio clandestino de Lima ingresan aves procedentes de los centros de acopio, que son beneficiadas para 
expendio directo al público consumidor. En algunos casos, el beneficio se practica en pequeña escala y con escasa bioseguridad (Zegarra et al., 2004). Algunos de estos centros de beneficio clandestino solo llegan hasta el desplumado, dejando el pollo entero para ser distribuido a los minoristas, en tanto que otros centros de beneficio llegan hasta la evisceración. En el presente estudio se seleccionaron al azar 17 centros de beneficio clandestino de aves en Lima Metropolitana, los cuales incluyeron ambos tipos de procesos de beneficio.

El procesamiento y análisis de las muestras se realizó en el Laboratorio de Salud Pública y Salud Ambiental de la Facultad de Medicina Veterinaria de la Universidad Nacional Mayor de San Marcos, Lima.

\section{Tamaño Muestral}

El número de aves por evaluar $(\mathrm{n}=164)$ se obtuvo mediante la fórmula para tamaño muestral de poblaciones infinitas (Daniel, 1996), usando un $97.5 \%$ de confianza y la proporción referencial de 20\% (Jiménez et $a l ., 2003)$ para Lima $(\mathrm{D}=0.07)$. En la práctica, se llegó a trabajar con 170 aves, tomándose 10 aves al azar de cada centro de beneficio.

\section{Obtención de Muestras}

El muestreo se realizó en horas de la madrugada, cuando se iniciaba el proceso de beneficio y donde la sensación térmica ambiental siempre era baja. Se colectaron muestras de superficie corporal mediante el método de enjuague. La canal eviscerada o sin eviscerar, dependiendo del tipo de proceso, era colocada en una bolsa plástica estéril con $400 \mathrm{ml}$ de agua peptonada al $0.1 \%$, se agitaba en forma vigorosa por un minuto y el líquido de enjuague era transferido a un envase estéril.

Además se colectaron muestras de heces mediante hisopado cloacal. Los hisopados fueron realizados después del desplume para ambos tipos de procesos y siempre antes de obtener la muestra del enjuagado de canal (Fig. 1). Los hisopos fueron colocados en tubos de ensayo con $10 \mathrm{ml}$ de agua peptonada al 0.1\% (Kanashiro et al., 2005).

\section{Aislamiento de Salmonella spp}

El proceso de aislamiento microbiológico se realizó según lo descrito por el ICMSF, USDA y por el método de referencia descrito en la norma técnica para productos cárnicos del INDECOPI (ICMSF, 1988; INDECOPI, 1998; USDA, 2011). Para el preenriquecimiento del líquido de enjuague se tomó $30 \mathrm{ml}$ de la muestra, se mezcló con 30 $\mathrm{ml}$ de agua peptonada al $0.1 \%$ y se incubó a $35{ }^{\circ} \mathrm{C}$ por $24 \mathrm{~h}$. Las muestras de hisopado también fueron incubadas a $35^{\circ} \mathrm{C}$ por $24 \mathrm{~h}$. Para el enriquecimiento de las muestras, se tomaron dos alícuotas de $1 \mathrm{ml}$ de cada muestra y se transfirieron a tubos con $10 \mathrm{ml}$ de Caldo Tetrationato y con $10 \mathrm{ml}$ de Caldo Selenito-Cistina, incubándose a $43^{\circ} \mathrm{C}$ por $24 \mathrm{~h}$.

Muestras de cada medio de enriquecimiento fueron sembradas mediante la técnica de agotamiento en Agar Sulfito Bismuto y Agar Verde Brillante, e incubadas a $35{ }^{\circ} \mathrm{C}$ por $24 \mathrm{~h}$. Las colonias resultantes se clasificaron en negativas o sospechosas a Salmonella spp según el siguiente criterio: las colonias en Agar Sulfito Bismuto se consideraron sospechosas si presentaron el centro negro, borde claro, con precipitado negro con o sin brillo metálico alrededor de las colonias (ICMSF, 1988); las colonias en Agar Verde Brillante se consideraron sospechosas si eran de color rosa translúcidas a opacas cuando se le comparó con el color rosa a rojo del medio (ICMSF, 1988).

\section{Identificación Bioquímica}

Las colonias sospechosas de Salmonella spp al cultivo microbiológico fueron sembradas en Agar Triple Azúcar Hierro (TSI), Agar Lisina Hierro (LIA), Caldo Úrea y Medio Sulfuro Indol Motilidad (SIM), y llevadas a incubación a $35^{\circ} \mathrm{C}$ por $24 \mathrm{~h}$, y se 


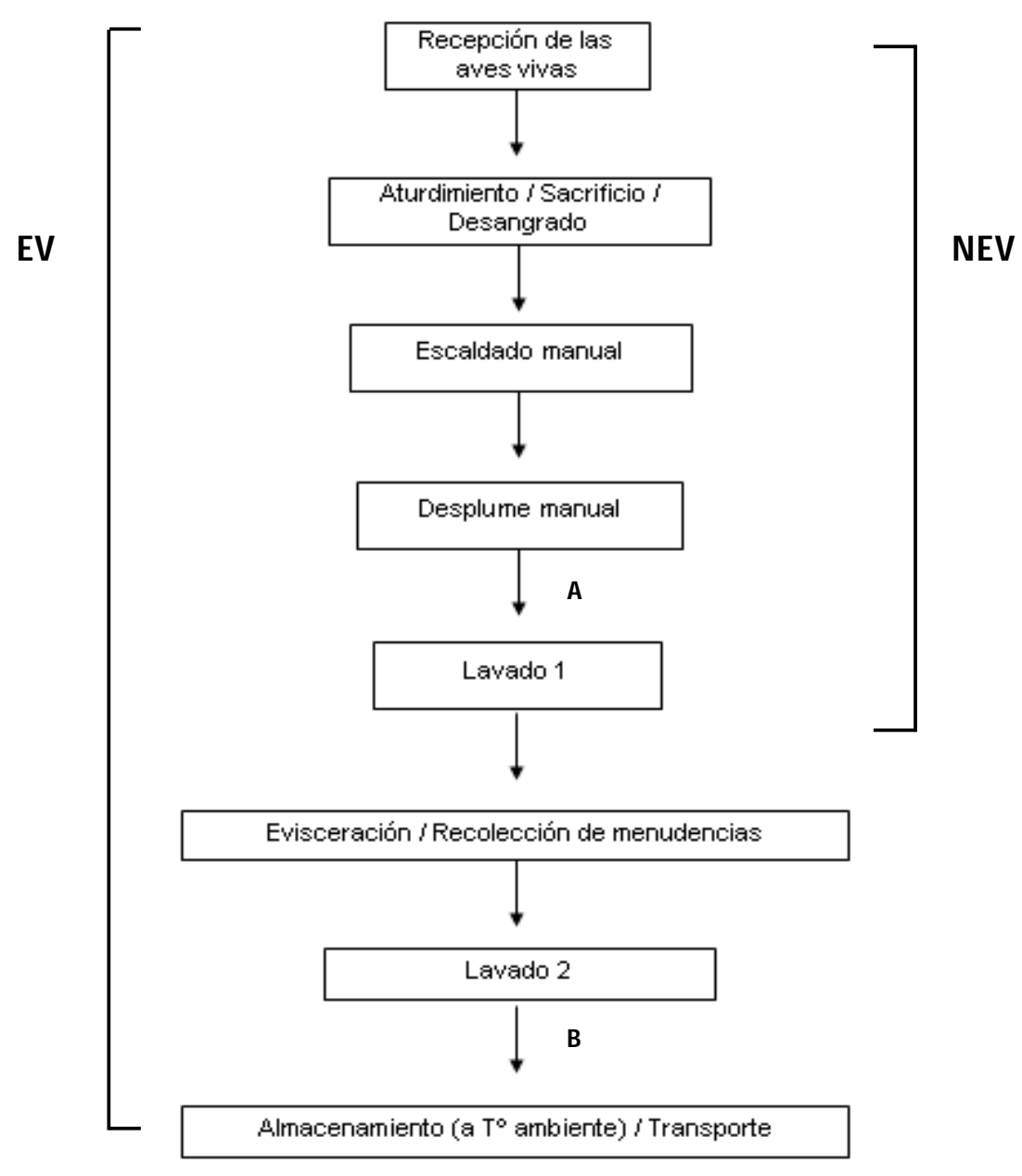

Figura 1. El proceso de beneficio en centros de beneficio clandestinos (peladurías) visitados. Se señala las fases del proceso de beneficio aplicadas para la obtención de aves evisceradas (EV) y no evisceradas (NEV). Se indican los puntos donde se realizó el muestreo: A) de obtención de hisopados, y de enjuague de superficie de canales no evisceradas, y B) de obtención de enjuegue de superficie de canales evisceradas

consideraron como negativas o sospechosas de Salmonella spp según los criterios de cada prueba detallados por el ICMSF (1988). Los cultivos sospechosos se sometieron a confirmación serológica, y aquellos que resultaron negativos en alguna de las pruebas bioquímicas volvieron a ser procesadas.

\section{Confirmación Serológica}

Las cepas que presentaron un perfil bioquímico compatible con Salmonella spp fueron confirmadas mediante la prueba de aglutinación en placa con Antisuero Polivalente Somático O Difco ${ }^{\mathrm{TM}}$ (Salmonella O Antiserum Poly A-I \& Vi, 222641). Según lo indicado por la norma técnica peruana, cualquier grado de aglutinación fue considerado como positivo a Salmonella spp (INDECOPI, 1998).

\section{Análisis Estadístico}

Los resultados se presentan como frecuencias y en porcentajes aplicando la fórmula descrita por Thursfield (1990), donde la 
Cuadro 1. Frecuencia de muestras positivas a Salmonella spp, según el tipo de centro de beneficio clandestino y de muestra

\begin{tabular}{|c|c|c|c|c|c|}
\hline \multicolumn{2}{|c|}{$\begin{array}{l}\text { Centro de beneficio } \\
\text { clandestino }\end{array}$} & \multicolumn{2}{|c|}{ Muestras } & \multicolumn{2}{|c|}{ Muestras positivas } \\
\hline Evisceran & N. ${ }^{\circ}$ & Tipo & N. ${ }^{\circ}$ & $\mathrm{N} .^{\circ}$ & $\%$ \\
\hline \multirow{3}{*}{ No } & \multirow{2}{*}{16} & $\begin{array}{l}\text { Superficie } \\
\text { corporal }\end{array}$ & 80 & 17 & 21.3 \\
\hline & & $\begin{array}{l}\text { Hisopado } \\
\text { cloacal }\end{array}$ & 80 & 23 & 28.8 \\
\hline & & & 160 & 40 & 25.0 \\
\hline \multirow{3}{*}{ Sí } & \multirow{2}{*}{18} & $\begin{array}{l}\text { Superficie } \\
\text { corporal }\end{array}$ & 90 & 23 & 25.6 \\
\hline & & $\begin{array}{l}\text { Hisopado } \\
\text { cloacal }\end{array}$ & 90 & 32 & 35.6 \\
\hline & \multicolumn{2}{|c|}{ Total } & 180 & 55 & 30.6 \\
\hline
\end{tabular}

prevalencia es igual al número de muestras positivas entre el total de muestras analizadas. Se evaluó el grado de concordancia entre las muestras tomadas de la superficie de la canal y las muestras de hisopado cloacal mediante el coeficiente de Kappa. Asimismo, se utilizó la prueba de Chi cuadrado para determinar la posible influencia del eviscerado sobre la presencia de Salmonella spp en la superficie corporal de las aves. Se utilizó el paquete estadístico SPSS v. 12 para Windows.

\section{Resultados}

En el Cuadro 1 se muestra la frecuencia de Salmonella spp en los ocho centros de beneficio clandestino donde el proceso finaliza con el desplumado y en los nueve centros donde el proceso finaliza con el eviscerado del ave. En ambos casos, el mayor número de aves positivas se encontró en las muestras de hisopado cloacal. Asimismo, la prueba de Kappa demostró la ausencia de concordancia en los resultados entre ambos tipos de muestras (Cuadros 2 y 3 ).
La prueba de Chi cuadrado indicó que independientemente de realizarse o no realizarse el eviscerado, no hubo diferencias significativas entre las frecuencias de muestras positivas $\left(\mathrm{X}^{2}=0.436\right)$.

\section{Discusión}

Las frecuencias obtenidas para Salmonella de 25.6 y $21.3 \%$ para superficie corporal en pollos enteros con y sin eviscerado fueron similares a los hallazgos en pechugas de pollo en expendio en mercados del distrito de Barranco, Lima (Jiménez et al., 2003). Asimismo, estos valores concuerdan con otros reportes de América Latina (Morillo et al., 1996); Pérez et al., 2008; WHO-GFN, 2008; Fonseca et al., 2010; Molina et al., 2010) y Australia (Fearnley et al., 2011).

En el presente trabajo se evaluó las canales de aves no evisceradas, cuya presentación es la más popular al momento de comercializar la carne de pollo en mercados locales, tiendas y puestos de expendio menor en el Perú. El consumidor asocia esta pre- 
Cuadro 2. Tabla de contingencia de las variables superficie corporal e hisopado cloacal en los centros de beneficio clandestino de aves don de no se realizaba el eviscerado

\begin{tabular}{|c|c|c|c|c|}
\hline & & \multicolumn{2}{|c|}{ Hisopado cloacal } & \multirow{2}{*}{ Total $(\%)$} \\
\hline & & Negativo (\%) & Positivo (\%) & \\
\hline \multirow{2}{*}{$\begin{array}{l}\text { Superficie } \\
\text { corporal }\end{array}$} & Negativo & $46(57.5)$ & $17(21.3)$ & $63(78.8)$ \\
\hline & Positivo & $11(13.7)$ & $6(7.5)$ & $17(21.2)$ \\
\hline \multicolumn{2}{|c|}{ Total (\%) } & $57(71.3)$ & $23(28.8)$ & $80(100.0)$ \\
\hline
\end{tabular}

Cuadro 3. Tabla de contingencia de las variables superficie corporal e hisopado cloacal en los centros de beneficio clandestino de aves donde se realizaba eviscerado

\begin{tabular}{ccccc}
\hline & & \multicolumn{2}{c}{ Hisopado cloacal } & \multirow{2}{*}{ Total (\%) } \\
\cline { 3 - 4 } & & Negativo (\%) & Positivo (\%) & \\
\hline \multirow{2}{*}{$\begin{array}{c}\text { Superficie } \\
\text { corporal }\end{array}$} & Negativo & $46(51.1)$ & $21(23.3)$ & $67(74.4)$ \\
\hline \multicolumn{2}{c}{ Total $(\%)$} & $12(13.3)$ & $11(12.2)$ & $23(25.5)$ \\
\hline \multicolumn{2}{c}{ Positivo } & $58(64.4)$ & $32(35.5)$ & $90(100.0)$ \\
\hline
\end{tabular}

sentación con la frescura del producto. Además, algunas vísceras se venden por separado para la preparación de ciertos platos tradicionales.

Se ha demostrado que los puntos críticos de la contaminación de la carne de pollo durante su beneficio se encuentran en los procesos de eviscerado y desplumado (FAO y OMS, 2003), ya que las canales de aves se contaminan al entrar en contacto con las heces provenientes de los intestinos o de las plumas sucias (Ponsa, 2005; Mastroeni y Maskell, 2006). Otra fuente de contaminación es el agua utilizada en el lavado de las canales, cuando esta se encuentra contenida en envases o pozas de lavado (OMS, 1988; $\mathrm{CCFH}, 2007)$, a lo cual se suma la inadecuada temperatura del agua de escaldado
(Mosquera et al., 2007) y la práctica de acumular los pollos enteros sin eviscerar en los bidones o tanques de lavado sin agua corriente.

En general, en los centros clandestinos visitados se observó las deficiencias antes mencionadas, lo que explica la presencia de Salmonella spp en las canales de pollos. La falta de utilización de buenas prácticas de manipulación así como la falta de higiene por parte de los trabajadores en estos centros de beneficio aumentan el riesgo de contaminación (SENASA, 2007). Además, en el proceso de beneficio de estos establecimientos no se incluyen actividades que reduzcan el riesgo de presentación de agentes patógenos en canales de aves, como puede ser el enfriado después del lavado. 
Los resultados obtenidos para hisopado cloacal de presencia de Salmonella spp son semejantes a otros reportes (Dione et al., 2009) obtenidos en pollos al final de la etapa de crecimiento. Incluso, se ha encontrado una frecuencia mayor de Salmonella en heces de pollos broilers durante el transporte hacia el matadero (Marin y Lainez, 2009). En el presente estudio, la Salmonella encontrada en el hisopado cloacal podría deberse a la contaminación ambiental de la explotación, o al estrés e inmunosupresión sufrida por las aves durante el transporte que altera la población microbiana del intestino (OMS, 1988).

La Salmonella no forma parte de la flora intestinal de las aves, y las aves la adquieren a partir de insectos, roedores, aves silvestres y el humano, así como por ingestión de alimento contaminado (Pérez et al., 2008). En el estrés del transporte se produce una gran multiplicación de bacterias intestinales, y la material fecal conteniendo Salmonella llega a contaminar la canal durante el faenado (Adelantado et al., 2008).

La falta de concordancia entre Salmonella spp encontrada en la superficie corporal y en el hisopado de cloaca, tanto en las canales evisceradas como no evisceradas $(\mathrm{k}<1)$, indica que hubo una importante cantidad de muestras que resultaron positivas a una prueba pero no a la otra. Por tanto, las pruebas no son reemplazables, debiéndose tomar ambos tipos de muestras para indicar si una canal de pollo es positiva o negativa a la contaminación por Salmonella spp.

\section{Conclusiones}

- $\quad$ El 23.5\% de las muestras de superficie corporal y el $32.4 \%$ de muestras de hisopado cloacal en aves de centros de beneficio clandestino de la ciudad de Lima fueron positivas a Salmonella spp.

- La práctica del eviscerado no influyó en la frecuencia de canales contaminadas con Salmonella spp.
- Para determinar si un ave es positiva o negativa a la presencia de Salmonella spp se deben tomar muestras de superficie corporal y de hisopado cloacal.

\section{Literatura Citada}

1. Acha PN, Szyfres B. 2003. Zoonosis y enfermedades transmisibles comunes al hombre y a los animales: Bacteriosis y micosis. $3^{\circ}$ ed. Washington: Organización Panamericana de la Salud. 398 p.

2. Adelantado C, Arosema E, Calvo M, Manteca L, Martín M, Ordóñez G, Ponsa F, et al. 2008. La Salmonella, de actualidad desde siempre. Barcelona: Real Escuela de Avicultura. 240 p.

3. [CCFH] Codex Committee on Food Higiene. 2007. Food safety risk profile for Salmonella species in broiler (young) chickens. Geneva: CCFH Working Group on Guidelines for control of Campylobacter and Salmonella spp in broiler (young bird) chicken meat. $30 \mathrm{p}$.

4. Daniel D. 1996. Bioestadística: Base para el análisis de las ciencias de la salud. $5^{\circ}$ ed. México DF: Limusa. 878 p.

5. Dione MM, Ieven M, Garin B, Marcotty T, Geerts S. 2009. Prevalence and antimicrobial resistance of Salmonella isolated from broiler farms, chicken carcasses, and street-vended restaurants in Casamance, Senegal. J Food Protect 72: 2423-2427.

6. [FAO, OMS] Food and Agriculture Organization of the United Nations, Organización Mundial de la Salud. 2003. Documento de debate sobre estrategias de gestión de riesgos de Salmonella spp en aves de corral. Orlando, EEUU: Comisión del Codex Alimentarius. $20 \mathrm{p}$.

7. Fearnley E, Raupach J, Lagala F, Cameron S. 2011. Salmonella in chicken meat, eggs and humans; Adelaide, South Australia, 2008. Int J Food Microbiol 146: 219-227. 
8. Fonseca G, Bernardino L, Quiñones E, Vázquez C. 2010. Comparación de la técnica de cultivo tradicional y la prueba rápida TecraTM para la detección de Salmonella spp en pollo crudo. En: Jornadas Científicas de Biomedicina y Biotecnología Molecular. Acapulco, México.

9. Guerrero R. 2007. Trazabilidad de la cadena de producción de carne de pollo en el Perú. Tesis de Magíster. Costa Rica: Universidad para la Cooperación Internacional. 84 p.

10. Gutiérrez A, Paasch L, Calderón N. 2008. Salmonelosis y campilobacteriosis, las zoonosis emergentes de mayor expansión en el mundo. Vet Méx 39: 81-90.

11. Heyndrickx M, Vandekerchove D, Herman L, Rollier I, Grijspeerdt K, De Zutter L. 2002. Routes for Salmonella contamination of poultry meat: epidemiological study from hatchery to slaughterhouse. Epidemiol Infect 129: 253-265.

12. [ICMSF] International Commission on Microbiological Specifications for Foods. 1988. Microorganisms in foods. Vol 1 «Detección de Salmonella». Microorganisms in foods $1.2^{\circ} \mathrm{ed}$. University of Toronto. p 160-172.

13. [INDECOPI] Instituto Nacional de Defensa de la Competencia y de la Protección de la Propiedad Intelectual. 1998. Norma Técnica Peruana 201.036. Carne y Productos Cárnicos: Método de referencia para la detección de Salmonella. $2^{\circ}$ ed. Lima: INDECOPI-CRT. $36 \mathrm{p}$.

14. Jiménez G, Getaz L, Malca E, Prada A. 2003. Aislamiento de enteropatógenos en carne de pollo que se expende en mercados y supermercados de Lima Metropolitana. En: XIV Jornada Científica "Raúl León Barúa". Lima: Universidad Peruana Cayetano Heredia.

15. Kanashiro A, Stoppa G, Cardoso A, Tessari E, Castro A. 2005. Serovars of Salmonella spp isolated from broiler chickens and commercial breeders in diverse regions in Brazil from July 1997 to December 2004. Braz J Poultry Sci 7: 195-198.

16. Málaga A. 2011. Plantas de beneficio peruanas: Hora Cero. Actualidad Avipecuaria. [Internet], [6 enero 2011]. Disponible en: http://www.actua-lidadavipecuaria.com/articulos/plantas-de-beneficio-peruanas-hora-cero.html

17. Marin C, Lainez M. 2009. Salmonella detection in feces during broiler rearing and after live transport to the slaughterhouse. Poultry Sci 88: 19992005.

18. Mastroeni P, Maskell D. 2006. Salmonella infections: clinical, immunological, and molecular aspects. Vol 9. UK: Cambridge University. $381 \mathrm{p}$.

19. [MINAG] Ministerio de Agricultura. 2010. Industria Avícola: Junio 2010 [Internet], [04 agosto 2010]. Disponible en: www.minag.gob.pe

20. [MINSA, DIGESA] Ministerio de Salud, Dirección General de Salud Ambiental. 2008. Norma sanitaria que establece los criterios microbiológicos de calidad sanitaria e inocuidad para los alimentos y bebidas de consumo humano. Norma Técnica Sanitaria No 071-V.01. Diario El Peruano: 29 de agosto de 2008. Lima, Perú.

21. Molina N, Millán B, Araque M. 2010. Indicadores de calidad sanitaria y fenotipificación de Salmonella enterica aislada de pollo crudo comercializado en el área urbana de Mérida, Venezuela. Infectio 14: 174-185.

22. Morillo A, Rodríguez $S$, Infante $D$, Noguera C, León A, Herrera A, Valdillo $P$. 1996. Detección de Salmonella spp en alas y vísceras comestibles de pollo. Vet Trop 21: 49-58.

23. Mosquera S, Alemán C, Villada $\mathrm{H}$. 2007. Aplicación de principios HACCP en el sacrificio y beneficio de pollos. Facultad de Ciencias Agropecuarias 5(2): 9-19.

24. [OMS] Organización Mundial de la Salud. 1988. Control de la salmonelosis: Importancia de la higiene veterinaria y 
de los productos de origen animal. Ginebra: OMS. Series de Informes Técnicos $774.95 \mathrm{p}$.

25. Pérez CM, Sánchez MM, Henao S, Cardona-Castro NM. 2008. Estandarización y evaluación de dos pruebas de reacción en cadena de la polimerasa para el diagnóstico de Salmonella enterica subespecie enterica en huevos. Arch Med Vet 40: 235-242.

26. Ponsa F. 2005. Puntos críticos para el control de Salmonella y Campylobacter en la carne de pollo. En: Jornadas Profesionales de Avicultura de Carne. Valladolid, España: Real Escuela de Avicultura.

27. [SENASA] Servicio Nacional de Sanidad Agraria. 2007. Reglamento del sistema sanitario avícola. Decreto Supremo No 029-2007-AG. Diario El Peruano: 1 de noviembre de 2007. Lima, Perú.

28. Thrusfield M. 1990. Epidemiología veterinaria. Zaragoza: Acribia. 339 p.

29. [USDA] United States Department of Agriculture. 2011. Isolation and identification of Salmonella from meat, poultry and egg products. Microbiology Laboratory Guidebook 4.05. [Internet], [15 setiembre 2011]. Disponible en: http:// www.fsis.usda.gov/PDF/MLG_4_05.pdf

30. [WHO, GFN] World Health Organization, Global Foodborne Infections Network. 2008. Manual de procedimientos: diagnóstico y caracterización de Salmonella spp. [Internet], [21 febrero 2009]. Disponible en: http:// fos.panalimentos.org/g fn/ ManualesdeProcedimiento/tabid/783/

31. Zamudio ML, Meza A, Bailón H, Martinez-Urtaza J, Campos J. 2011. Experiencias en la vigilancia epidemiológica de agentes patógenos transmitidos por alimentos a través de electroforesis en campo pulsado (PFGE) en el Perú. Rev Per Med Exper Salud Púb 28: 128-135.

32. Zegarra J, Palomino L, Ramos D, Manzanedo R, Angulo C, Alvarado A. 2004. Clasificación y priorización de los departamentos del Perú según variables epidemiológicas en sanidad avícola: I etapa. En: XVII Congreso Nacional de Ciencias Veterinarias. Tacna: Colegio Médico Veterinario del Perú. 